\title{
The Case for Universal Testing of Colorectal Tumors for Microsatellite Instability: A Coming Mismatch Between Clinical and Laboratory Testing
}

\author{
Patrick M. Lynch ${ }^{1}$
}

Published online: 30 June 2015

(c) Springer Science+Business Media New York 2015

There is a clear trend toward universal testing of newly diagnosed colorectal cancers (CRCs) for evidence of microsatellite instability (MSI) [1-4]. MSI involves easily detected changes in the length of short nucleotide repeat sequences that are acquired in tumors but that are not present in normal tissues. Such changes, originally detected with PCR-based methods, are essentially a universal feature of CRCs in patients with hereditary nonpolyposis colorectal cancer (HNPCC or "Lynch syndrome"). However, about $15 \%$ of all CRCs show MSI, and of these, $20 \%$ have underlying HNPCC, with additional, but readily available tests needed to distinguish likely sporadic from likely HNPCC [5]. Convergent data support the feasibility and utility of universal MSI testing:

(a) Population studies have confirmed the above proportions of MSI and of HNPCC [5].

(b) These same studies have reported that a sizable proportion of cases of HNPCC would not likely have been detected using existing clinical selection tools such as the Amsterdam criteria or even the much more liberal Bethesda guidelines. Hence, the rationale for universal testing is supported, even for cases for which HNPCC is not suspected clinically.

(c) Immunohistochemistry (IHC) for mismatch repair (MMR) protein expression is a good, albeit not perfect, surrogate for polymerase chain reaction (PCR)-based MSI testing [6]. Its low cost, preliminary identification of the underlying gene that is

Patrick M. Lynch

plynch@mdanderson.org

1 Department of Gastroenterology, Hepatology, and Nutrition, The University of Texas MD Anderson Cancer Center, 1400 Pressler St., Unit 1466, Houston, TX 77030, USA likely mutated, and ready availability in most community surgical pathology facilities are advantages for using IHC.

(d) The costs of performing MSI testing, including methylation/BRAF testing to identify and exclude from further HNPCC consideration, have continued to fall.

(e) Panels of molecular tumor markers have been developed to provide more accurate prognosis and treatment response predictions [7]. Such panels can and do include markers of MSI and of methylation/ BRAF, providing information about the potential "HNPCC-ness" of a tumor even when such information was not specifically sought [8].

(f) A priori risk models, while providing a rationale for HNPCC germline mutation testing that does not require initial evaluation of tumors for MSI, have not been widely used as a foundation for such testing. Reasons include the above factors that favor tumorbased testing when tumor is available on the one hand, and the lack of usefulness of a negative germline test in cancer-free, at-risk subjects when no underlying mismatch repair gene mutation has already been found in the family.

Concurrent with these efforts, investigators have sought to make sense of it all by considering the yield and the cost-effectiveness of numerous strategies for evaluating tumors. In the paper by Kidambi et al. published in this issue of Digestive Diseases and Sciences [9], a recent (2007-2010) historical cohort of CRCs studied at a single academic institution that was evaluated according to prevalent clinical selection criteria was compared with another cohort (2007-2013) in which all cases underwent molecular characterization by means of IHC for MMR 
gene expression. Clinical selection was based on age $(<50)$, characteristic "MSI histology" in those aged 50-60, or any patient with an additional HNPCC spectrum tumor. Unlike the Bethesda guidelines, a family history of cancer was not a selection criterion. In keeping with a commonly employed approach, the University of California, San Francisco group used IHC for screening, with BRAF mutation testing specified for cases with loss of MutL homolog 1 (MLH1) expression, in order to exclude likely sporadic cases whose loss of protein expression was likely due to epigenetic silencing of MLH1. BRAF mutation testing is commonly employed as a surrogate for MLH1 methylation assays as BRAF mutations are virtually never seen in tumors from patients with underlying MMR mutations.

The key findings from this study were that while nearly half $(42 \%)$ of those subjected to universal screening would not have been evaluated had the "selective" criteria been applied, only one of the eight cases identified under the universal testing strategy would not have been screened under the selective strategy. One could argue that IHC is a bit less sensitive than PCR-based MSI testing and thus might have missed some cases that would have led to an HNPCC diagnosis. Nevertheless, there is no good reason to think that the yield of such a marginally more sensitive assay would have selectively favored the universal testing group over the clinically selected group.

Older patients, particularly those with a negative family history, are likely to have MSI on the basis of epigenetic silencing of MLH1. More recent data suggest that there are other cases, involving loss of $\mathrm{MSH} 2$ for example, in which epigenetic events may account for the MSI and loss of protein expression [9, 10]. Heretofore, and in contradistinction to the situation with MLH1 loss, such cases had been assumed to have underlying germline mutations, even when such mutations could not be detected with sequencing and methods to detect deletions and other rearrangements. Such findings do at least provide some reassurance that there are plausible alternative explanations for lack of mutation detection, especially in those cases with no antecedent family history, the typical situation in which "MSI but no mutation detected" is found.

Returning the issue addressed in the Kidambi et al. [9] paper, their findings do provide support for those who contend that universal testing is not cost-effective, at least not insofar as HNPCC case finding is concerned. There are, however, other arguments that can be made in favor of universal testing. Perhaps the most simplistic one is that testing all incident CRCs removes major variables. It is possible that the decision by Kidambi et al. to rely on criteria other than family history in developing even the clinical selection strategy reflects the challenges in properly collecting and analyzing such information on a routine basis. If all cases are screened with MSI and/or IHC, one need not be concerned about these issues.

Other similarly constructed studies have yielded data more clearly supportive of universal testing [11]. But even if the present study is correct in concluding that universal testing does not really detect more cases of HNPCC than a clinically directed approach, the tide of events may well be favoring a non-discriminating approach anyway. As noted above, the use of panels of molecular markers is on the rise. Whether on a very comprehensive research basis or through the use of clinical panels, markers of interest in detecting HNPCC are nested with the much broader list of molecular tumor markers. One example is the "Foundation 1 " panel a proprietary panel that can test for alterations in a host of genes that have been more or less implicated in etiologic, prognostic, or therapeutic patterns in diverse tumors, including those of the colorectum. These proceed from already existing targeted molecular approaches and from a comprehensive catalog of mutations from The Cancer Genome Atlas or TCGA, a comprehensive library of mutations discerned in various tumors through the use of advanced molecular sequencing methodologies.

In light of this trend, it may simply not matter whether a clinical strategy is helpful for selecting cases for further molecular characterization for purposes of HNPCC detection. Sooner or later, probably sooner, tumors will be routinely subjected to routine and comprehensive molecular characterization. I conducted an informal poll of gastrointestinal medical oncologists at my institution, posing the single question of "Do you think panel testing of tumors will be routine and universal for essentially all stage CRCs within the next 5 years?" All stated this was likely or highly likely. The emphasis under this paradigm will shift more and more to the informatics of sorting out what mutations are relevant for what purpose-genetic basis, prognosis, therapeutics, or other. In the case of possible genetic susceptibility, the interpretive challenges have been present for some time. As noted, when MSI is detected and/ or when MLH1 expression is lost, there is the task of excluding "false positives" through BRAF mutation and/or methylation assays. One can anticipate an ever more complex and at the same time iterative approach when broader panels are involved.

\section{References}

1. Beamer LC, Grant ML, Espenschied CR, et al. Reflex immunohistochemistry and microsatellite instability testing of colorectal tumors for Lynch syndrome among US cancer programs and follow-up of abnormal results. J Clin Oncol. 2012;30:1058-1063. doi:10.1200/JCO.2011.38.4719.

2. Kastrinos F, Syngal S. Screening patients with colorectal cancer for Lynch syndrome: what are we waiting for? J Clin Oncol. 2012;30:1024-1027. doi:10.1200/JCO.2011.40.7171. 
3. Evaluation of Genomic Applications in Practice and Prevention (EGAPP) Working Group. Recommendations from the EGAPP Working Group: genetic testing strategies in newly diagnosed individuals with colorectal cancer aimed at reducing morbidity and mortality from Lynch syndrome in relatives. Genet Med. 2009;11:35-41. doi:10.1097/GIM.0b013e31818fa2ff.

4. Palomaki GE, McClain MR, Melillo S, Hampel HL, Thibodeau SN. EGAPP supplementary evidence review: DNA testing strategies aimed at reducing morbidity and mortality from Lynch syndrome. Genet Med. 2009;11:42-65. doi:10.1097/GIM.0b013e $31818 \mathrm{fa} 2 \mathrm{db}$.

5. Hampel H, Frankel WL, Martin E, et al. Screening for the Lynch syndrome (hereditary nonpolyposis colorectal cancer). $N$ Engl $J$ Med. 2005;352:1851-1860. doi:10.1056/NEJMoa043146.

6. Lindor NM, Burgart LJ, Leontovich $\mathrm{O}$, et al. Immunohistochemistry versus microsatellite instability testing in phenotyping colorectal tumors. J Clin Oncol. 2002;20:1043-1048.

7. Frampton GM, Fichtenholtz A, Otto GA, et al. Development and validation of a clinical cancer genomic profiling test based on massively parallel DNA sequencing. Nat Biotechnol. 2013;31:1023-1031. doi:10.1038/nbt.2696.

8. Parsons DW, Roy A, Plon SE, Roychowdhury S, Chinnaiyan AM. Clinical tumor sequencing: an incidental casualty of the American College of Medical Genetics and Genomics recommendations for reporting of incidental findings. J Clin Oncol. 2014;32:2203-2205. doi:10.1200/JCO.2013.54.8917.

9. Kidambi TD, Blanco A, Myers M, Conrad P, Loranger K, Terdiman JP. Selective versus universal screening for lynch syndrome: a six-year clinical experience. Dig Dis Sci. (Epub ahead of print). doi:10.1007/s10620-014-3234-z.

10. Carethers JM. Differentiating Lynch-like from Lynch syndrome. Gastroenterology. 2014;146:602-604. doi:10.1053/j.gastro.2014. 01.041.

11. Mensenkamp AR, Vogelaar IP, van Zelst-Stams WA, et al. Somatic mutations in MLH1 and MSH2 are a frequent cause of mismatch-repair deficiency in Lynch syndrome-like tumors. Gastroenterology. 2014;146:643-646. doi:10.1053/j.gastro.2013. 12.002 . 